\title{
THIN FILM PC-EL TYPE ELECTRIC AND OPTICAL SIGNAL DELAYING SYSTEMS
}

\author{
Z. PORADA \\ Institute of Engineering and Electronics, Technical University, Warszawska 24, 31-155 \\ Kraków (Poland) \\ E. SCHABOWSKA-OSIOWSKA \\ Institute of Electronics, Academy of Mining and Metallurgy, Al.Mickiewicza 30, 30-159 \\ Kraków (Poland)
}

(Received February 24, 1994; in final form April 13, 1994)

\section{INTRODUCTION}

The rapid development of optoelectronics and more widespread utilization in technology of various types of optoelectronic systems has resulted in an increase in interest in thin film PC-EL type systems. They are increasingly being used as logic circuits, as amplifiers of weak light signals, and can be used to delay electric and optical signals. The electric and optical signal delay lines find application in various types of industrial telecommunication systems.

In the presented work, the dependence of the delay times of electric pulse signals and optical signals in the form of rectangular light pulses on the parameters of PCEL system was investigated. Characteristics obtained were compared with the curves calculated from a proposed mathematical model.

\section{THEORETICAL CONSIDERATION}

A diagram of electric signal delaying system is shown in Fig. 1. In such a system, the input signal is the voltage $U_{\text {inp }}$ supplying the element $E L_{1}$, and the output signal is the current $I_{\text {out }}$ through the element PC.

The switching on of the voltage $U_{\text {inp }}$ will cause the emission of light from the element $\mathrm{EL}_{1}$ of luminance $\mathrm{B}_{1}$, which can be described ${ }^{1}$ by the relation:

$$
B_{1}(t)=B_{01} \exp \left(-\gamma_{1} t\right) \exp \left[b_{1} /\left(\left|U_{\text {inp }}\right|\right)^{1 / 2}\right]
$$

where $B_{01}, \gamma_{1}$ and $b_{1}$ are the parameters, constant for given electroluminescent cell $\mathrm{EL}_{1}$. 


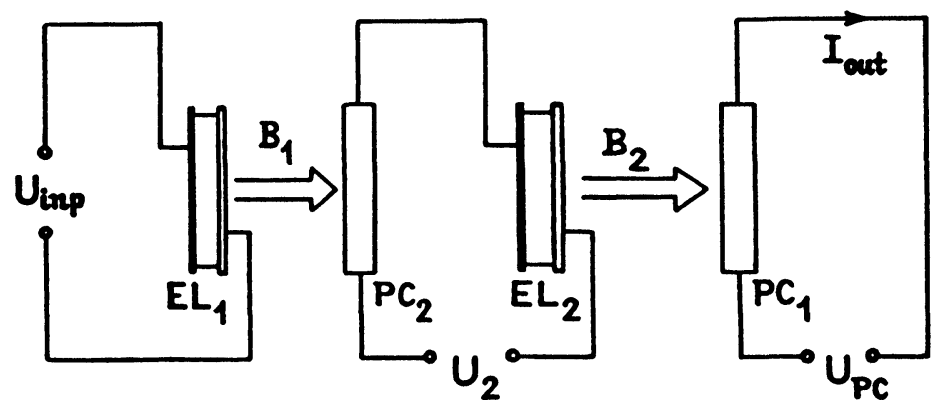

FIGURE 1 PC-EL type electric signal delaying system.

The light with luminance $\mathrm{B}_{1}$ will then illuminate the element $\mathrm{PC}_{2}$, causing an increase of its conductance $\mathrm{G}_{\mathrm{PC} 2}$, which can be described ${ }^{2}$ by following equation:

$\frac{\mathrm{d}}{\mathrm{dt}} \mathrm{G}_{\mathrm{PC} 2}=\mathrm{a}_{2} \beta_{1} \mathrm{~B}_{1}-\frac{\mathrm{G}_{\mathrm{PC} 2}-\mathrm{G}_{02}}{\tau_{2}}$

where $\mathrm{G}_{02}$ is the "dark" conductance of non-illuminated element $\mathrm{PC}_{2}, \tau_{2}$ is the photoconductivity rise time for this element, $\mathrm{a}_{2}$ is a parameter, constant for given element $\mathrm{PC}_{2}$, and $\beta_{1}$ is the unidirectional optical coupling coefficient of the element $\mathrm{EL}_{1}$ with the element $\mathrm{PC}_{2}$.

The increase of the conductance $\mathrm{G}_{\mathrm{PC} 2}$ will cause an increase of the voltage on the element $E L_{2}$, so that it will emit the light of luminance $B_{2}$, which can be described with a formula similar as in the case of luminance $B_{1}$ :

$B_{2}(t)=B_{02} \exp \left(-\gamma_{2} t\right) \exp \left[-b_{2} /\left(\left|U_{E L 2}\right|\right)^{1 / 2}\right]$

where the voltage $\mathrm{U}_{\mathrm{EL} 2}$ on the element $\mathrm{EL}_{2}$ is given by the formula:

$\mathrm{C}_{\mathrm{EL} 2} \frac{\mathrm{d}}{\mathrm{dt}} \mathrm{U}_{\mathrm{EL} 2}+\mathrm{U}_{\mathrm{EL} 2}\left(\mathrm{G}_{\mathrm{EL} 2}+\mathrm{G}_{\mathrm{PC} 2}\right)=\mathrm{G}_{\mathrm{PC} 2} \mathrm{U}_{2}$

where $B_{02}, \gamma_{2}$, and $b_{2}$ are parameters of electroluminescent cell $E L_{2}, C_{E L 2}$ is its capacity, $G_{E L 2}$ is its leakage conductance, and $U_{2}$ is the voltage supplying the $P_{2^{-}}$ $\mathrm{EL}_{2}$ system.

The light emitted by the element $\mathrm{EL}_{2}$ illuminates the photoconducting element $P_{1}$, causing an increase of its conductance $G_{P C l}$ and thereby an increase in the output current $I_{\text {out }}$. The course of this current vs. time $I_{\text {out }}(t)$ can be expressed by the formula:

$\mathrm{I}_{\text {out }}(\mathrm{t})=\mathrm{G}_{\mathrm{PCl}}(\mathrm{t}) \mathrm{U}_{1}$

where the conductance $\mathrm{G}_{\mathrm{PCl}}(\mathrm{t})$ is described by the eqution:

$\frac{d}{d t} G_{P C 1}=a_{1} \beta_{2} B_{2}-\frac{G_{P C 1}-G_{01}}{\tau_{1}}$ 


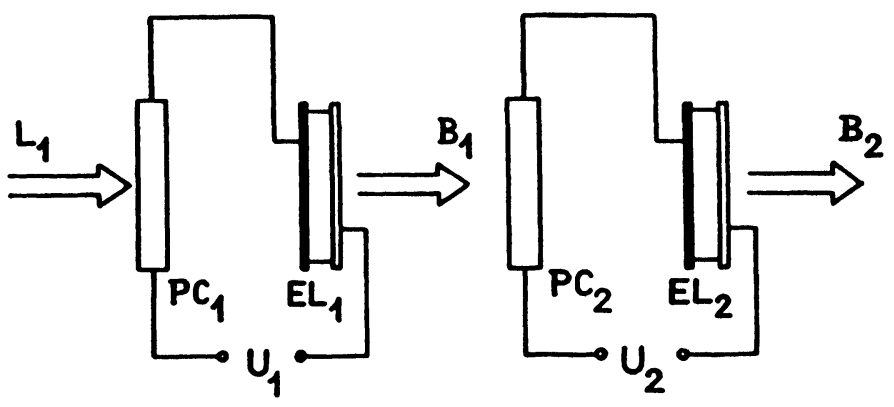

FIGURE 2 Diagram of an optical signal delaying PC-EL system.

where $G_{01}$ is the "dark" conductance of non-illuminated element $P_{1}, \tau_{1}$ is the photoconductivity rise time for this element, $\mathrm{a}_{1}$ is a parameter, constant for given element $\mathrm{PC}_{1}, \boldsymbol{\beta}_{2}$ is the unidirectional optical coupling coefficient of the element $E_{2}$ with the element $P C_{1}$, and $U_{1}$ is the voltage supplying the circuit with the element $\mathrm{PC}_{1}$.

The solution of the set of equations (1)-(6) allows one to calculate the delay time $\tau_{2}$ of the output signal $I_{\text {out }}$ in relation to the input signal $U_{\text {inp }}$.

In Fig. 2, the diagram of an optical signal delaying PC-EL system is shown. In the circuit under consideration, the input signal is a light pulse illuminating $\mathbf{P C}_{1}$ element, the illumination of which is $\mathrm{L}_{1}$, and the output signal is the light emitted by the element $E L_{2}$, the luminance of which is $B_{2}$.

The illumination of the element $\mathrm{PC}_{1}$ causes an increase in the conductance $\mathrm{G}_{\mathrm{PCl}}$ of this element, the result of which the voltage on the element $E L_{1}$ will increase so that the electroluminescent cell $\mathrm{EL}_{1}$ will emit the light of luminance $\mathrm{B}_{1}$. This luminance can be described by the expression (1), where $U_{i n p}=U_{E L 1}$. The voltage $\mathrm{U}_{\mathrm{ELI}}$ is given by the formula:

$C_{E L 1} \frac{d}{d t} U_{E L 1}+U_{E L I}\left(G_{E L 1}+G_{P C l}\right)=G_{P C I} U_{1}$

where $\mathrm{C}_{\mathrm{ELI}}$ are, respectively, the capacity and the leakage conductance of the element $E L_{1}, U_{1}$ is the voltage supplying the $P_{1}-E L_{1}$ circuit, and the conductance $\mathrm{G}_{\mathrm{PCl}}$ is described by the formula:

$\frac{d}{d t} G_{P C l}=a_{1} L_{1}-\frac{G_{P C l}-G_{01}}{\tau_{1}}$

where $\mathrm{a}_{1}$ is a parameter, constant for the element $\mathrm{PC}_{1}, \tau_{1}$ is the photoconductivity rise time for this element, and $G_{01}$ is the "dark" conductance of non-illuminated element $\mathrm{PC}_{1}$.

As can be seen on Fig. 3, the element $\mathrm{EL}_{1}$ is optically coupled with the element $\mathrm{PC}_{2}$ and, thereby, the illumination of the element $\mathrm{PC}_{2}$ will be $\mathrm{L}_{2}=\beta \mathrm{B}_{1}$, where $\beta$ is the unidirectional optical coupling coefficient. Thus, the output signal in the 


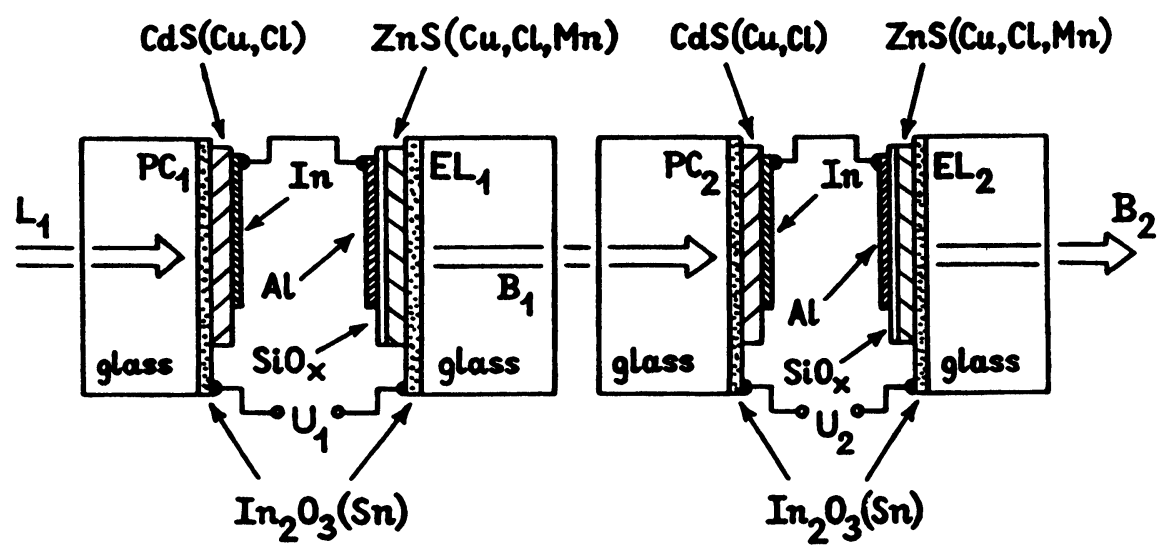

FIGURE 3 Thin film PC-EL type optical signal delaying circuit.

form of luminance $B_{2}$ of the light emitted by the element $\mathrm{EL}_{2}$ can be described using the dependence:

$B_{2}(t)=B_{02} \exp \left(-\gamma_{2} t\right) \exp \left[-b_{2} /\left(\left|U_{E L 2}\right|\right)^{1 / 2}\right]$

where the voltage $\mathrm{U}_{\mathrm{EL} 2}$ is described by the formula:

$\mathrm{C}_{\mathrm{EL} 1} \frac{\mathrm{d}}{\mathrm{dt}} \mathrm{U}_{\mathrm{EL} 2}+\mathrm{U}_{\mathrm{EL} 2}\left(\mathrm{G}_{\mathrm{EL} 2}+\mathrm{G}_{\mathrm{PC}}\right)=\mathrm{G}_{\mathrm{PC} 2} \mathrm{U}_{2}$

and the conductance $\mathrm{G}_{\mathrm{PC} 2}$ of the element $\mathrm{PC}_{2}$ is given by the equation:

$\frac{d}{d t} G_{P C 2}=a_{2} \beta B_{1}-\frac{G_{P C 2}-G_{02}}{\tau_{2}}$

where $B_{02}, \gamma_{2}$, and $b_{2}$ are parameters, constant for given element $E_{2}, C_{E L 2}$ is the electric capacity of this element, $G_{E L 2}$ is its leakage conductance, $a_{2}$ is a parameter, constant for the element $\mathrm{PC}_{2}, \mathrm{G}_{02}$ is the "dark" conductance of non-illuminated element $P_{2}$, and $U_{2}$ is the voltage supplying the $P_{2}-E L_{2}$ circuit.

After solving the set of equations (7)-(11) the delay time $\tau_{\mathrm{op}}$ of output signal $B_{2}$ in relation to input signal $L_{1}$ can be determined.

\section{RESULTS OF EXPERIMENTAL INVESTIGATION}

The circuit PC-EL delaying the optical signal (Fig. 3) was composed of two photoconducting elements, $\mathrm{PC}_{1}$ and $\mathrm{PC}_{2}$, and two electroluminescent cells, $\mathrm{EL}_{1}$ and $\mathrm{EL}_{2}$.

In the elements PC, the photoconducting layer was made of copper- and chlorinedoped cadmium sulphide, the transparent electrode was a tin-doped indium oxide layer, and the second electrode an indium layer ${ }^{3}$. 

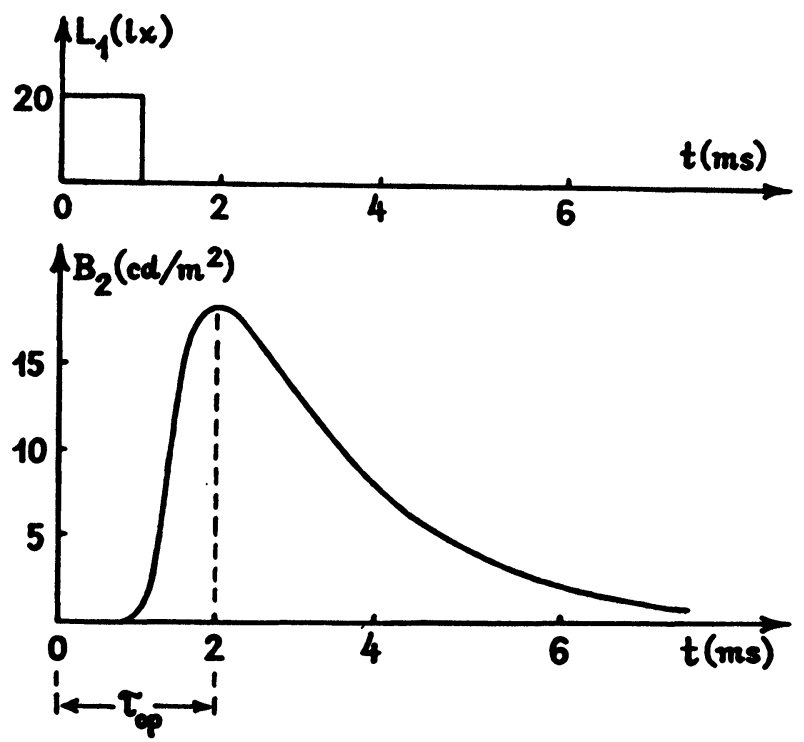

FIGURE 4 The dependence of luminance $B_{2}$ and illumination $L_{1}$ on time for the delaying circuit PCEL supplied with equal sinusoidal voltages $U_{1}=U_{2}=420 \mathrm{~V}$ of the frequency $f=70 \mathrm{~Hz}$.

In the elements EL, the electroluminescent layer was copper-, chlorine- and manganese-doped zinc sulphide, the transparent electrode was a tin-doped indium oxide layer, and the second electrode was an aluminium layer ${ }^{4}$.

In the circuit under consideration, the dependence of luminance $\mathrm{B}_{2}$ on time, as well as the dependence of the illumination $\mathrm{L}_{1}$ on time, for the delaying circuit PCEL supplied with equal sinusoidal voltages $U_{1}$ and $U_{2}\left(f_{1}=f_{2}=f\right)$ of the frequency $\mathrm{f}=70 \mathrm{~Hz}$ (Fig. 4) were investigated.

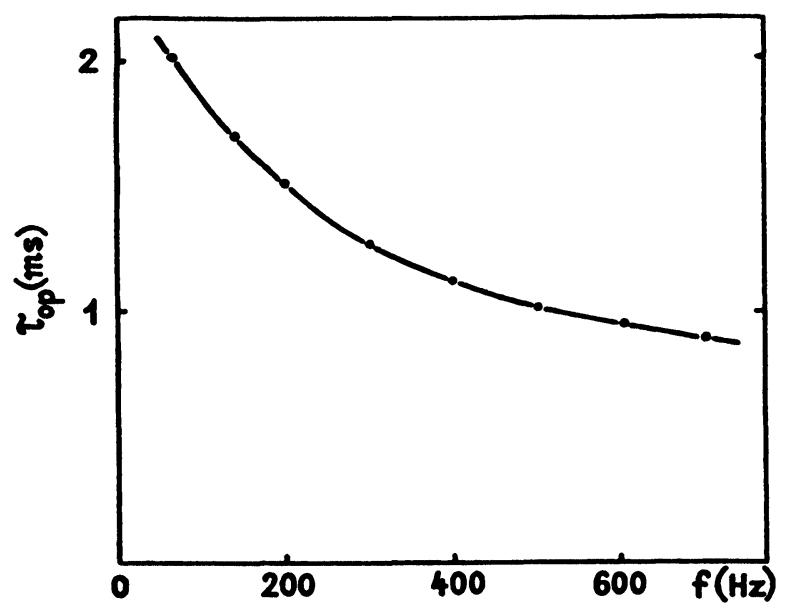

FIGURE 5 The dependence of the delay time $\tau_{\mathrm{op}}$ on the frequency of the voltage supplying the PCEL circuit. 


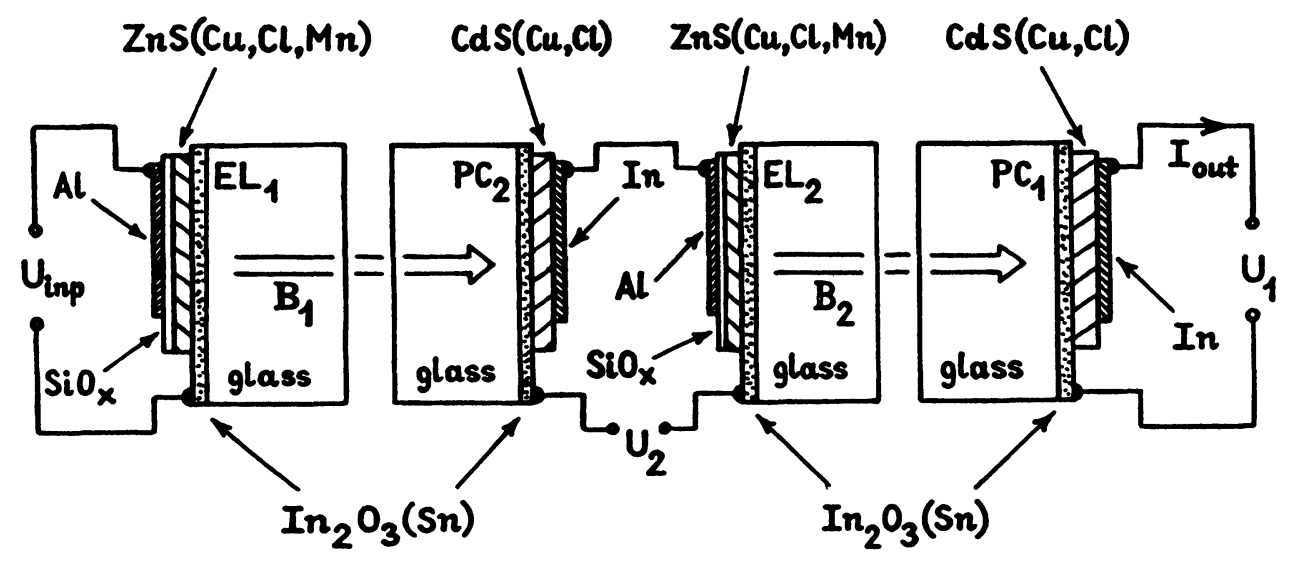

FIGURE 6 Thin film PC-EL type electric signal delaying circuit.

In Fig. 5, the dependence of the delay time $\tau_{\mathrm{op}}$ on the frequency of the voltage supplying the PC-EL circuit $\left(f_{1}=f_{2}=f\right)$ is presented. The electric signal delaying circuit is shown in Fig. 6 . In the considered circuit, the dependence of the voltage $\mathrm{U}_{\text {inp }}$ and the current $\mathrm{I}_{\text {out }}$ on time was investigated (Fig. 7). In Fig. 8, the dependence of delay time $\tau_{0}$ on the frequency of voltage supplying the $\mathrm{PC}_{2}-\mathrm{EL}_{2}$ circuit is presented.

\section{CONCLUSIONS}

Results from the analysis of proposed theoretical model indicate that the delay time of the optical signal $\tau_{\mathrm{op}}$ depends, in the first place, on the photoconductivity

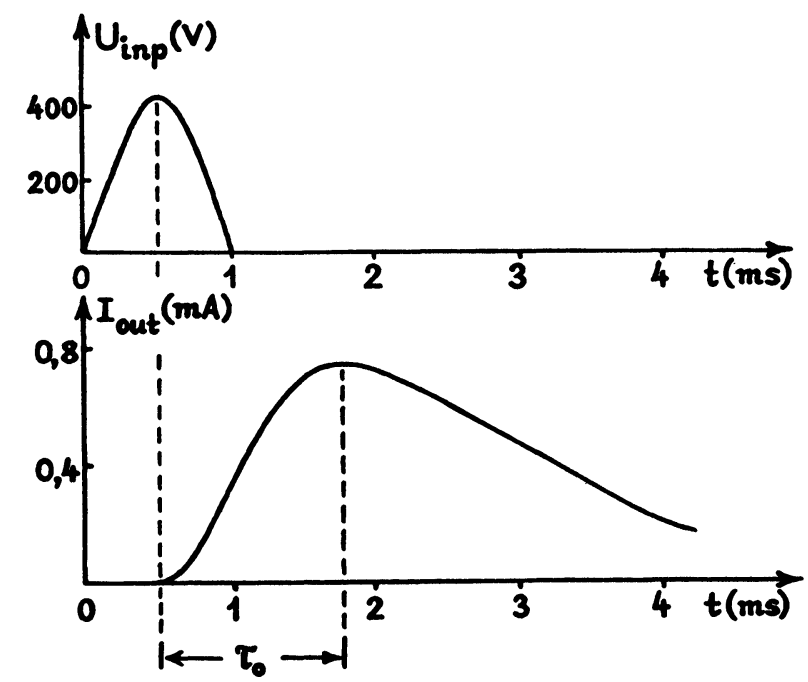

FIGURE 7 Time dependences of the input signal $U_{\text {inp }}$ and of the output signal $I_{\text {out }} ; f=140 \mathrm{~Hz}$. 


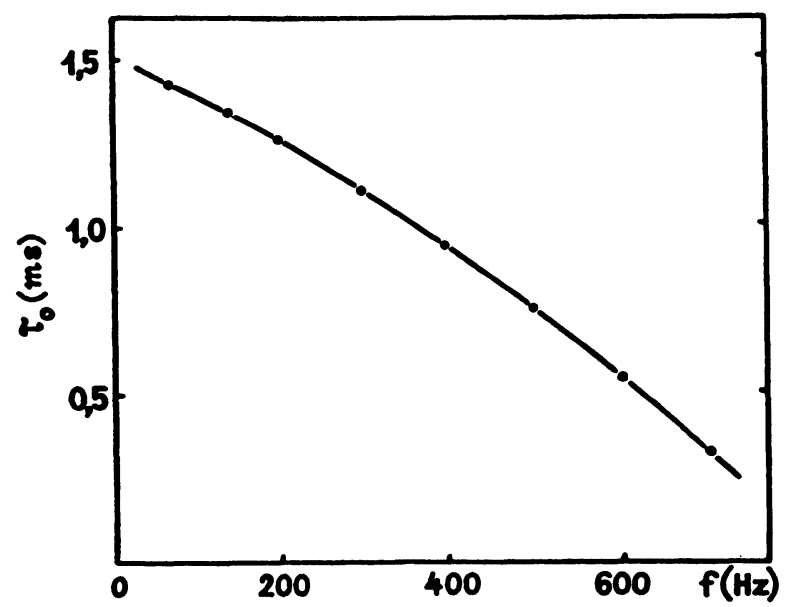

FIGURE 8 Dependence of delay time $\tau_{1}$ on the frequency of supplying voltage $U_{2}$.

rise times $\tau_{1}$ and $\tau_{2}$, as well as on the frequency of supplying voltages $U_{1}$ and $U_{2}$; the value $\tau_{\mathrm{op}}$ can be increased, in the circuit under consideration, by using a greater number of $\mathrm{PC}_{1}-\mathrm{EL}_{2}$ circuits.

The delay time of electric signal $\tau_{0}$ depends also on the values of photoconductivity rise times $\tau_{1}$ and $\tau_{2}$, as well as on the frequency $f$ of voltage $U_{2}$ supplying the $\mathrm{PC}_{2}-\mathrm{EL}_{2}$ circuit.

The PC-EL type electric signals delaying systems, as well as optical delaying system, have an additional advantage; namely, the connection of input circuits with output circuits in these systems is not galvanic, i.e., from the point of view of electric circuits they perform the function of a separator.

\section{REFERENCES}

1. Z. Porada and E. Schabowska, J. Luminescence, 21, 129 (1980).

2. P.S. Kireev, Semiconductor Physics, Mir, Moscow, 1978 (English translation).

3. Z. Porada and E. Schabowska-Osiowska, Active and Passive Elec. Comp., 13, 151 (1988).

4. Z. Porada and E. Schabowska, Vacuum, 33, 179 (1983). 

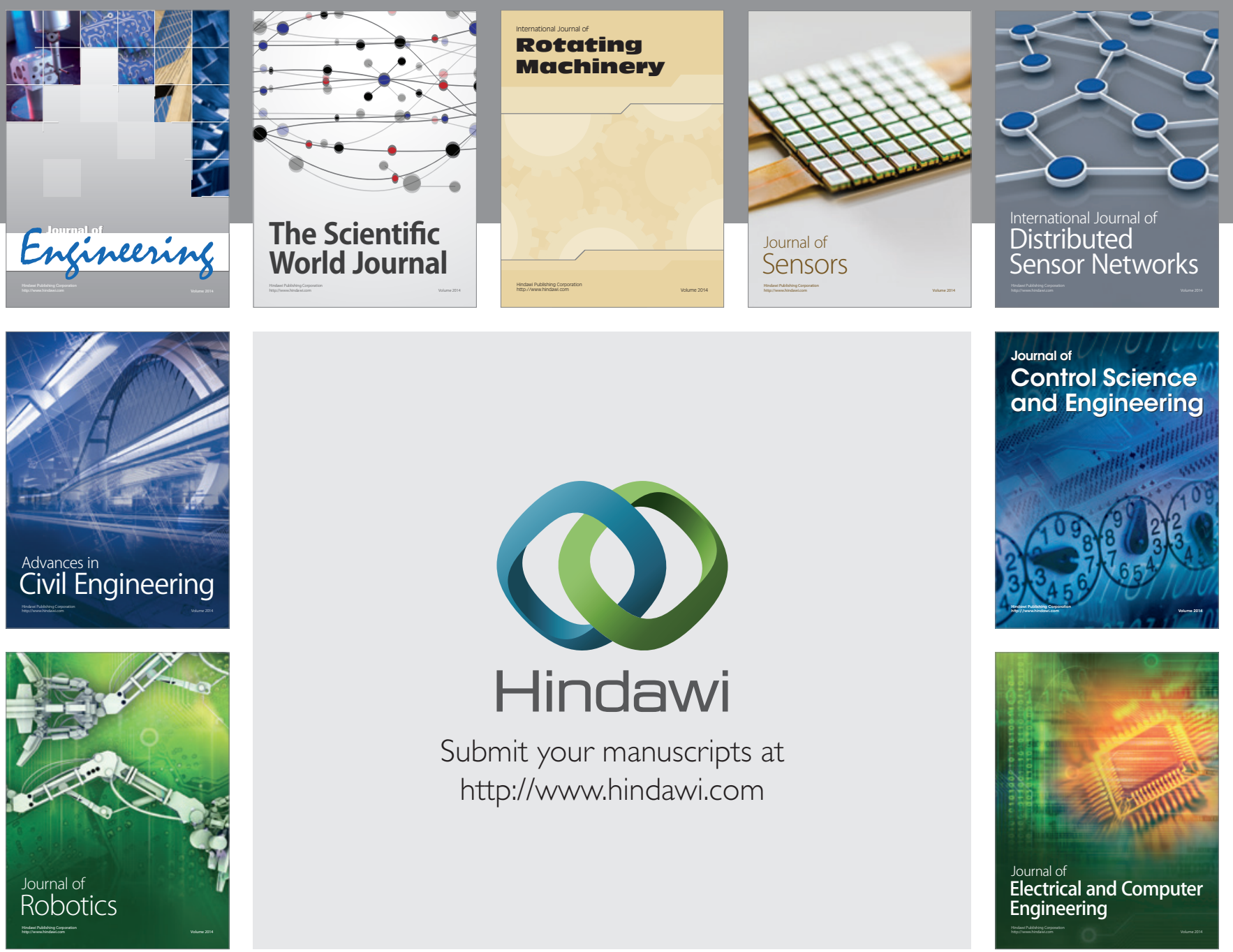

Submit your manuscripts at

http://www.hindawi.com
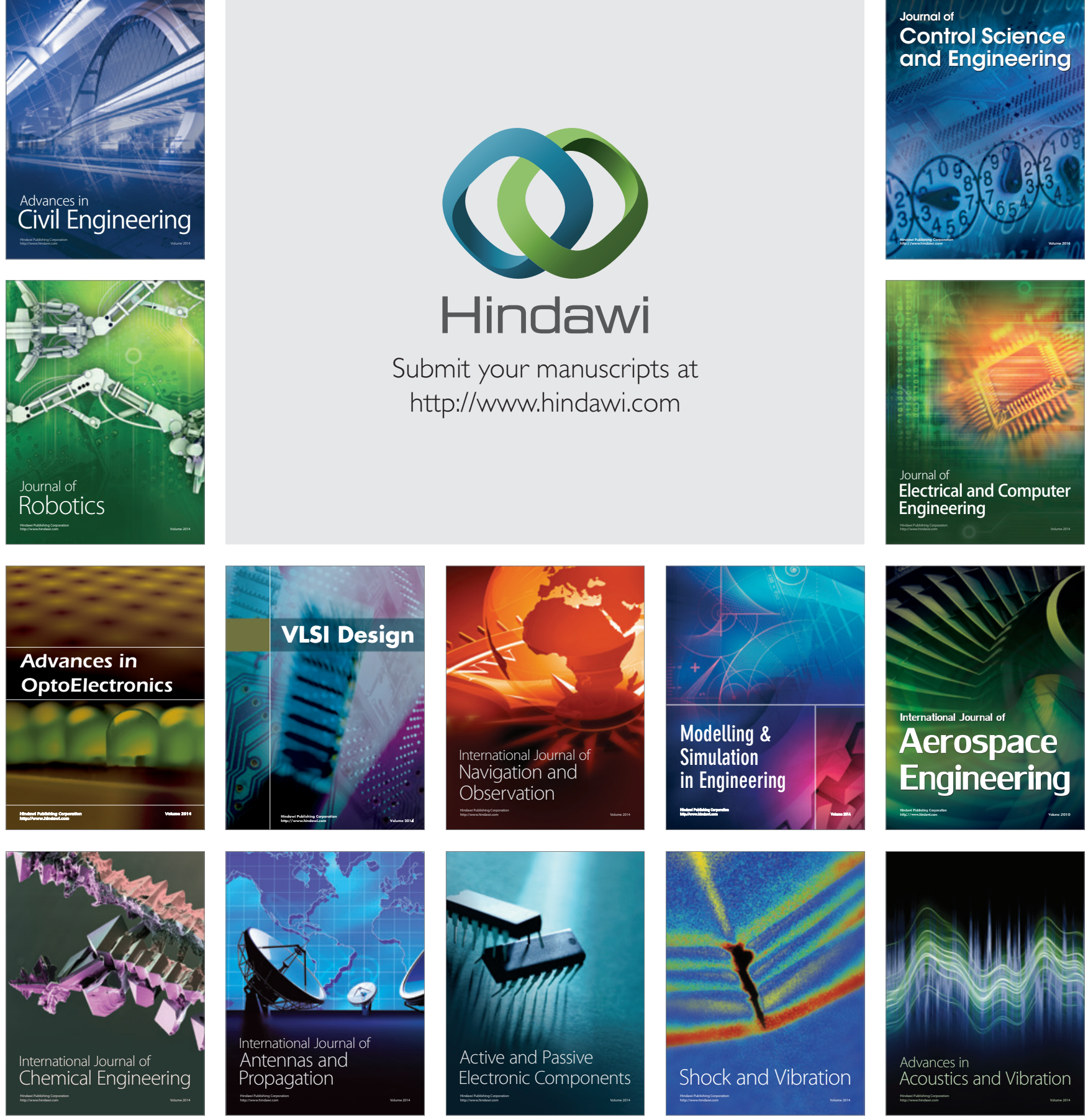